\title{
Role of TGF- $\beta 1 /$ Smad3 Signaling Pathway in Secretion of Type I and III Collagen by Vascular Smooth Muscle Cells of Rats Undergoing Balloon Injury
}

\author{
Ping Lu, Songhao Wang, Wenwei Cai, and Jing Sheng \\ Department of Geriatrics, The Ninth People's Hospital, Shanghai Jiaotong University School of Medicine, No. 639, Zhizaoju Road, \\ Shanghai 200011, China \\ Correspondence should be addressed to Jing Sheng, jenniferberry06@hotmail.com
}

Received 28 April 2012; Revised 25 June 2012; Accepted 25 June 2012

Academic Editor: Richard Tucker

Copyright () 2012 Ping Lu et al. This is an open access article distributed under the Creative Commons Attribution License, which permits unrestricted use, distribution, and reproduction in any medium, provided the original work is properly cited.

\begin{abstract}
Antisense Smad3 adenoviral vectors were used to transfect vascular smooth muscle cells (VSMCs) from rats with balloon injury or infused into the rat balloon-catheter injured carotid arteries, and the role of TGF- $\beta 1 / \mathrm{Smad} 3$ signaling pathway in the secretion of type I and III collagen by VSMCs following balloon injury was investigated. Antisense Smad3 adenoviral vectors were used to transfect these VSMCs (antisense Smad3 group). A total 90 rats were randomly assigned into blank control group, experiment group, negative control group. In the in vitro study, the expression of type I and III collagen was markedly reduced in the antisense Smad3 group when compared with the control groups $(P<0.05)$. In the in vivo study, the expression of type I and III collagen was significantly lower than that in the negative control group at 3 days, 1 week and 2 weeks after injury $(P<0.05)$. At 2 weeks and 3 months after injury, the lumen area in the antisense Smad3 group was markedly increased but the intimal area dramatically reduced when compared with the negative control $(P<0.05)$. We conclude that transfection of VSMCs with antisense Smad 3 can reduce the secretion of type I and III collagen which then inhibit intimal hyperplasia.
\end{abstract}

\section{Introduction}

To date, percutaneous transluminal coronary angioplasty (PTCA) and stenting have been the important strategies for the treatment of coronary heart diseases (CHD). However, the incidence of restenosis (RS) is at a high level within 6 months after surgery [1]. Although the drug-eluting stent has significantly reduced the RS incidence, the application of drug-eluting stents still has some limitations. Thus, the prevention and treatment of RS have become a hot topic in the field of interventional cardiology.

PTCA may cause damage to the vascular endothelial cells (VECs), aggregation of inflammatory cells, activation of platelets, and release of some cytokines such as interleukin1 (IL-1), interferon (IFN), fibroblast growth factor (FGF), platelet-derived factor (PDGF), and transforming growth factor- $\beta$ (TGF- $\beta$ ) [2]. These can lead to the migration and hyperplasia of vascular smooth muscle cells (VSMCs) finally resulting in vascular stenosis [3]. TGF- $\beta$, with many biological activities, has been confirmed to be an important cytokine regulating the production and deposition of extracellular matrix (ECM) [4].

TGF- $\beta 1$ is a growth factor with hormone-like activity, has the molecular weight of about $25 \mathrm{kDa}$, and can form dimmers via disulfide bond. Studies showed, at $6 \mathrm{~h}$ after balloon injury, the TGF- $\beta 1$ expression began to rise and the increased TGF- $\beta 1$ sustained for 14 days [5]. TGF- $\beta 1$ may stimulate the proliferation of neointimal cells and induce the synthesis and remodeling of ECM resulting in vascular restenosis [6]. In vivo study has demonstrated that TGF- $\beta 1$ could promote the hyperplasia of neointima [7]. Tranilast was found to effectively reduce the thickening of neointima and restenosis at the stent via reducing the TGF- $\beta 1$ activity or directly inhibit TGF- $\beta 1$ expression $[8,9]$. In addition, TGF- $\beta 1$ was demonstrated to stimulate the synthesis of fibronectin in the VSMCs via the Smad3 signaling pathway, leading to the deposition of ECM in the neointima [10], 
which contributes to the RS. These findings demonstrate that neointimal formation is closely related to the TGF- $\beta 1 / \mathrm{Smad} 3$ signaling pathway, but the exact mechanism is still unclear.

In our previous study, the adenoviral vectors expressing antisense Smad3 were constructed and used to transfect VSMCs collected from rats undergoing vascular injury. Results revealed that inhibition of Smad3 expression could compromise the proliferation of VSMCs [11]. In the rat carotid artery balloon injury model, inhibition of Smad3 expression was found to reduce the thickening of vascular intima and decrease the ratio of intima area to media area $(\mathrm{I} / \mathrm{M})[12]$. In the present study, adenoviral vectors expressing antisense Smad3 were used to transfect VSMCs of rats undergoing balloon injury in vitro or directly injected into rats receiving balloon injury followed by detection of collagen I and III (components of ECM). This study aimed to investigate the role of TGF- $\beta 1 / \mathrm{Smad} 3$ signaling pathway in the neointimal formation and our findings may provide evidence for the prevention and treatment of postoperative RS.

\section{Materials and Methods}

2.1. Materials. Male Sprague-Dawley (SD) rats aged 6 weeks (weight: $350-450 \mathrm{~g}$ ) were purchased from the Shanghai Slac Laboratory Animal Co., Ltd (Production license: SCXK [Hu] 2004-0005; Use license: SYXK[Hu]2007-0007). The construction, packaging, amplification, and purification of blank adenovirus and adenovirus expressing antisense Smad3 [13] were purchased from the Shanghai Ji Kaiji Chemical Technology Company Limited (Shanghai, China). Adenoviral vectors were prepared at $6 \times 10 \mathrm{pfu} / \mathrm{mL}$ before study.

2.2. In Vitro Culture and Transfection of VSMCs from Rats Receiving Carotid Artery Balloon Injury. The 2F catheter was used to induce left carotid artery balloon injury in above rats according to previously reported methods [14]. Two weeks later, tissue block culture method was employed to culture the VSMCs in DMEM containing 10\% fetal bovine serum (FBS). Immunohistochemistry was done to identify the VSMCs. The VSMCs from injured artery (injury group) and normal VSMCs (normal group) were included. The cells of passage 3 were used in the following experiments.

In the in vitro experiment, the VSMCs from injured artery and divided into three groups and included: experiment group (cells transfected with adenovirus expressing antisense Smad3), negative control group (cells transfected with blank adenovirus), and blank control group (cells collected from rats with balloon injury but without any treatment). Transfection was performed with the multiplicity of infection (MOI) of $100 \mathrm{pfu}$, and detection was done $72 \mathrm{~h}$ later.

2.3. In Vivo Experiment and Sample Collection. The rat balloon injury model was established in 90 rats according to previously reported methods [14]. In the blank control group, rats underwent carotid artery balloon injury alone; in the experiment group, rats received carotid artery balloon injury and subsequently the adenovirus expressing antisense Smad3 $(0.5 \mathrm{~mL} ; 6 \times 10 \mathrm{pfu} / \mathrm{mL})$ was injected via the external carotid artery; in the negative control group, rats received carotid artery balloon injury, and subsequently the blank adenovirus solution $(0.5 \mathrm{~mL} ; 6 \times 10 \mathrm{pfu} / \mathrm{mL})$ was injected via the external carotid artery. During the injection, the tension of common carotid artery was maintained. At 30 min after perfusion, the external carotid artery was clamped but the blood flow of the internal and common carotid artery was assured. Following wound closure, the rats were given ad libitum assess to food and water.

At 1 day, 3 days, 1 week, 2 weeks, 1 month, and 3 months after balloon injury, rats were sacrificed. The left common carotid artery was collected, washed in normal saline, fixed in $4 \%$ paraformaldehyde, embedded in paraffin and sectioned. A fraction of tissues were stored in liquid nitrogen and then at $-80^{\circ} \mathrm{C}$ for real-time PCR. Before sample collection, $5 \mathrm{~mL}$ of blood was collected from the heart and centrifuged. The serum was obtained and stored at $-80^{\circ} \mathrm{C}$ for use.

2.4. Real-Time PCR. Total RNA was extracted by using Trizol reagent. Then, $2 \mu \mathrm{g}$ of RNA was used for reverse transcription into cDNA. Real-time PCR was done in the MX3000P thermal cycler (Applied Biosystems). The primers are shown in Table 1. The conditions for PCR were $95^{\circ} \mathrm{C}$ for $5 \mathrm{~min}$ and 40 cycles of $95^{\circ} \mathrm{C}$ for $30 \mathrm{~s}, 58^{\circ} \mathrm{C}$ for $30 \mathrm{~s}$, and $72^{\circ} \mathrm{C}$ for $45 \mathrm{~s}$. $\beta$-actin served as an internal reference and the expression of target genes was normalized to that of $\beta$-actin.

2.5. Detection with Enzyme Linked Immunosorbent Assay (ELISA). The contents of TGF- $\beta 1$ and type I and III collagen in the culture medium and serum were measured with ELISA according to manufacturer's instructions.

2.6. Pathological Examinations. The paraffin-embedded tissues were sectioned and HE staining was performed. The morphology of blood vessel wall was observed under a light microscope. Image analysis system (Image pro Plus 4.5) was used to measure the lumen area and intima area.

2.7. Statistical Analysis. All data were expressed as means \pm SD, and SPSS version 11.0 was employed for statistical analysis. One way analysis of variance was done to compare the means among different groups. A value of $P<0.05$ was considered statistically significant.

\section{Results}

3.1. Expression of TGF- $\beta 1$, Smad3, and Type I and III Collagen in VSMCs from Rats with Arterial Balloon Injury. Significant differences were found in the expressions of TGF- $\beta 1$, Smad3, type I and III collagen between the injury group and normal group $(P<0.05)$. Results from real-time PCR are shown in Figure 1(a). In the injury group, the mRNA expressions of TGF- $\beta 1$, Smad3, type I and III collagen were increased by $21 \%, 32 \%, 41 \%$, and $46 \%$, respectively, as compared to the normal group. Results from ELISA are shown in 
TABle 1: Primers for real-time PCR.

\begin{tabular}{lcc}
\hline Genes & \multicolumn{1}{c}{ Sequences } & Expected size \\
\hline TGF- $\beta 1$ & $\begin{array}{c}\text { Forward 5'-ACTCCCAGAAAAGCAAGCAA-3' } \\
\text { Reverse 5'-CGAGCAGGAATGAGAAGAGG-3' }\end{array}$ & 211 bp \\
\hline Smad3 & $\begin{array}{c}\text { Forward 5'-CATTACCATCCCCAGGTCAC-3' } \\
\text { Reverse 5'-CGTAACTCATGGTGGCTGTG-3' }\end{array}$ & $102 \mathrm{bp}$ \\
\hline Collagen I & $\begin{array}{c}\text { Forward 5'-CCC TGA AGT CAG CTG CAT-3' } \\
\text { Reverse 5'-ATA TTC TTC TGG GCA GAA-3' }\end{array}$ & $193 \mathrm{bp}$ \\
\hline Collagen III & $\begin{array}{c}\text { Forward 5'-TGGTCCTCAGGGTGTAAAGG-3' } \\
\text { Reverse 5'-GTCCAGCATCACCTTTTGG-3' }\end{array}$ & $232 \mathrm{bp}$ \\
\hline -actin & $\begin{array}{c}\text { Forward 5'-CTGTCCCTGTATGCCTCTG-3' } \\
\text { Reverse 5'-TGTCACGCACGATTTCC-3' }\end{array}$ & $217 \mathrm{bp}$ \\
\hline
\end{tabular}

Figure 1(b). Results demonstrated that the contents of TGF$\beta 1$, Smad3, and type I and III collagen in the injury group were $81 \pm 6.71,56 \pm 5.21,37 \pm 2.37$, and $77 \pm 6.13 \mathrm{ng} / \mathrm{mL}$, respectively, which were significantly different from those in the normal group.

3.2. Smad3 Expression in VSMCs Transfected with Adenovirus Expressing Antisense Smad3. In the in vitro study, the mRNA expression of Smad3 in the experiment group was markedly lowered by $47 \%$ when compared to the negative control group (Figure 2) $(P<0.01)$, but no marked difference was noted between the negative control group and blank control group $(P>0.05)$.

3.3. Type I and III Collagen Expression in VSMCs Transfected with Adenovirus Expressing Antisense Smad3. In the in vitro experiment, the type I and III collagen expression in the experiment group were dramatically reduced by $35 \%$ and $47 \%$, respectively, when compared with negative control group $(P<0.05)$ (Figure $3(\mathrm{a}))$. ELISA revealed the contents of type I and III collagen in the experiment group were $21 \pm$ $1.34 \mathrm{ng} / \mathrm{mL}$ and $55 \pm 2.74 \mathrm{ng} / \mathrm{mL}$, respectively, which were markedly lower than those in the negative control group $(P<0.05)$ (Figure 3(b)). However, significant differences in the contents of type I and III collagen were not found between the negative control group and the blank control group $(P>0.05)$.

3.4. Expression of Type I and III Collagen In Vivo. Previous study showed the Smad3 expression in the experiment group was significantly reduced at 1 day, 3 days, and 2 weeks after injury as compared to the negative control group $(P<0.05)$, thereafter decreased gradually and reached a minimal level at 3 months after injury $(0.96 \pm 0.24)$ [12]. No significant difference in the Smad3 expression was found between the experiment group and the negative control group at 3 months after injury $(P>0.05)$. In the present study, the type I and III collagen was also measured in vivo. Our findings revealed the type I collagen expression was dramatically inhibited at 3 days, 1 week and 2 weeks after injury when compared with the negative control group $(P<0.05)$ (Figures $4(\mathrm{a})-4(\mathrm{~b}))$, but there was no significant difference between experiment group and negative control group at 3 months after injury $(P>0.05)$. In addition, the type III collagen expression was dramatically reduced at 3 days, 1 week, and 2 weeks after injury when compared with the negative control group $(P<0.05)$ (Figures 4 (c)$4(\mathrm{~d}))$, but no pronounced difference was found between the experiment group and the negative control group at 3 months after injury $(P>0.05)$.

3.5. Effect of In Vivo Transfection on Vascular Lumen. HE staining was performed to observe the morphology of blood vessels. A previous study showed the ratio of $\mathrm{I} / \mathrm{M}$ in the experiment group was markedly lower than that in the negative control group $(P<0.05)$ at 1 day, 2 weeks, and 3 months after injury, but there was no significant difference between the negative control group and the blank control group at above three time points $(P>0.05)$ [12]. In the present study, we also measured the vascular lumen area and intima area and results are shown in Table 2. Results showed the lumen area at 2 weeks and 3 months after injury in the experiment group was significantly increased as compared to the negative control group $(P<0.05)$, but marked difference was absent at 1 week after injury $(P>0.05)$. The intima area in the injury group was dramatically reduced at 2 weeks and 3 months after injury as compared to the negative control group, but no significant difference was found between them at 1 week after injury $(P>0.05)$.

\section{Discussion}

Percutaneous coronary intervention (PCI) has been the most effective strategy in the treatment of CHD, but the incidence of RS is as high as $35-50 \%$ at 3-6 months after injury [15], which has been a pivotal factor affecting the therapeutic efficacy of PCI and become a focus in the field of CHD. Overdeposition of ECM is a key step in the vascular intimal hyperplasia and lumen stenosis. In humans, VSMCs account for only $11 \%$ of neointima during the RS and the remaining components are ECM [16]. Currently, studies have demonstrated that inhibition of ECM deposition is a novel strategy for the prevention of post-PTCA restenosis [17]. TGF- $\beta 1$ can promote the proliferation and migration 
TABLE 2: Vascular lumen area and intima area in different groups.

\begin{tabular}{lcccccc}
\hline & \multicolumn{3}{c}{ Lumen area $\left(\mathrm{mm}^{2}\right)$} & \multicolumn{3}{c}{ Intima area $\left(\mathrm{mm}^{2}\right)$} \\
& $1 \mathrm{w}$ & $2 \mathrm{w}$ & $3 \mathrm{~m}$ & $1 \mathrm{w}$ & $2 \mathrm{w}$ & $3 \mathrm{~m}$ \\
\hline Blank control & $0.39 \pm 0.05$ & $0.31 \pm 0.03$ & $0.14 \pm 0.05$ & $0.07 \pm 0.01$ & $0.12 \pm 0.04$ & $0.39 \pm 0.03$ \\
Negative control & $0.40 \pm 0.07$ & $0.33 \pm 0.03$ & $0.17 \pm 0.02$ & $0.08 \pm 0.02$ & $0.13 \pm 0.02$ & $0.42 \pm 0.01$ \\
Antisense Smad3 & $0.40 \pm 0.08$ & $0.40 \pm 0.06$ & $0.21 \pm 0.03 \mathbf{\Delta}$ & $0.06 \pm 0.02$ & $0.07 \pm 0.01 \mathbf{0}$ & $0.17 \pm 0.04$ \\
\hline
\end{tabular}

Note: $\boldsymbol{\Delta} P<0.05$ and $P<0.01$ versus negative control.

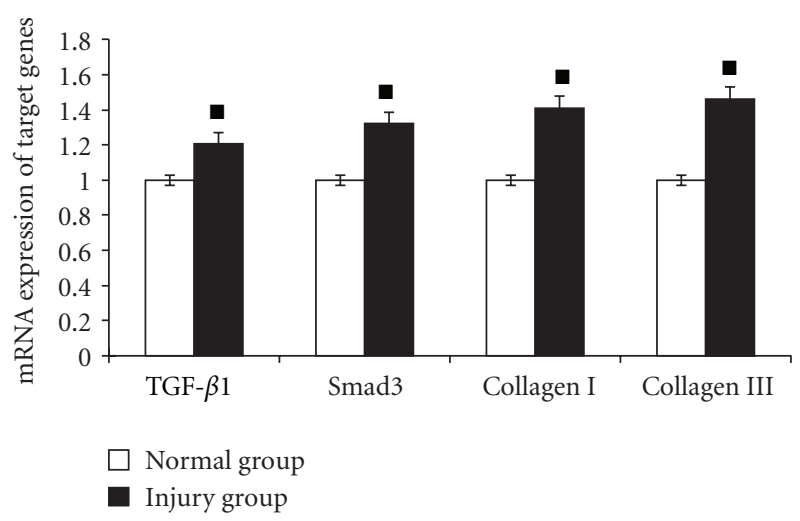

(a)

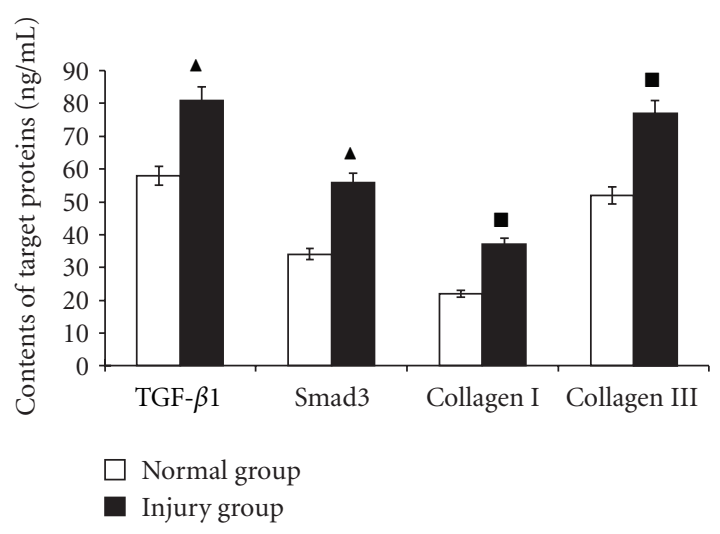

(b)

FIGURE 1: Expressions of TGF- $\beta$, Smad3, and type I and III collagen in VSMCs from rats undergoing carotid artery balloon injury. SD rats aged 6 weeks were employed to establish the postcarotid artery balloon injury RS model. Two weeks after injury, tissue block culture was performed to culture the VSMCs in DMEM containing $10 \%$ FBS. These cells were divided into normal group and injury group, and immunohistochemistry for $\alpha$-SMA was done to identify the VSMCs. VSMCs of passage 3 were collected for the following experiments. (a) quantitative RT-PCR. The mRNA expressions of TGF- $\beta 1$, Smad3, and type I and III collagen were independently normalized to that of $\beta$-actin as the relative expression. The mRNA expression of target genes in the normal group was defined as the baseline level, and that in the injury group was normalized to that in the normal group as fold change which was then used for delineation. Data were expressed as mean \pm SD. (b) The culture medium was collected, and ELISA was performed to measure the contents of TGF- $\beta 1$, type I and III collagen. ${ }^{\wedge} P<0.05,{ }^{-} P<0.01$ versus normal group.

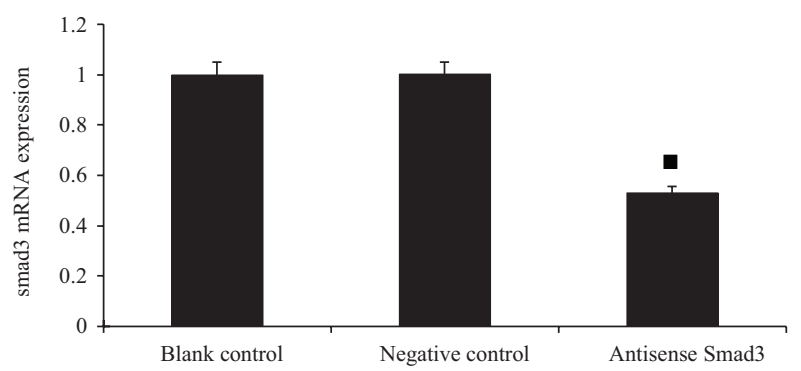

FIGURE 2: Smad3 expression in VSMCs transfected with adenovirus expressing antisense Smad3 in vitro. Following injury, VSMCs were collected and divided into 3 groups. In the antisense Smad3 group, cells were transfected with adenovirus expressing antisense Smad3; in the negative control group, cells were transfected with blank adenovirus; in the blank control group, cells received no treatment. Transfection was done with adenovirus at an MOI of $100 \mathrm{pfu}$. Three days later, total RNA was extracted from each group and RT-PCR was performed. The mRNA expression of Smad3 was normalized to that of $\beta$-actin. The mRNA expression of Smad 3 in the blank control group served as the baseline level, and the mRNA expression of Smad3 in other groups was normalized to that in the blank control group as the fold change which was used for delineation. Data were expressed as mean $\pm \mathrm{SD}$. $\Delta P<0.05, \boldsymbol{\square}_{P}<0.01$ versus negative control group.

of SMCs in the vascular intima and facilitate the formation of ECM resulting in RS [4].

TGF- $\beta 1$ can bind to receptors on the smooth muscle and fibroblasts and then exert its biological effects via inducing the phosphorylation of signal regulatory proteins. The down-stream proteins of TGF- $\beta 1$ include ERK, MAPK, JNK, and Smad3 [6], and Smad3 has been confirmed as a definite and important regulator at the down-stream of TGF$\beta 1[18,19]$. Following vascular injury, the TGF- $\beta 1$ is upregulated [20] and then stimulates the fibronectin synthesis via the Smad3 signaling pathway, which may increase the deposition of ECM in the neointima [10]. In addition, TGF$\beta 1$ can induce the transformation of fibroblasts into $\alpha$-SMA expressing myofibroblasts [21] and promote the secretion of ECM, which have been found to play important roles in the vascular remodeling.

Members of Smad family play critical roles in the TGF$\beta$ signal transduction and different Smads mediate the signal transduction of different members of Smad family. Studies showed the gene transcription and apoptosis could be regulated by TGF- $\beta / S \operatorname{Smad} 2 / 3$-Smad4 [22]. In the SMCs, 


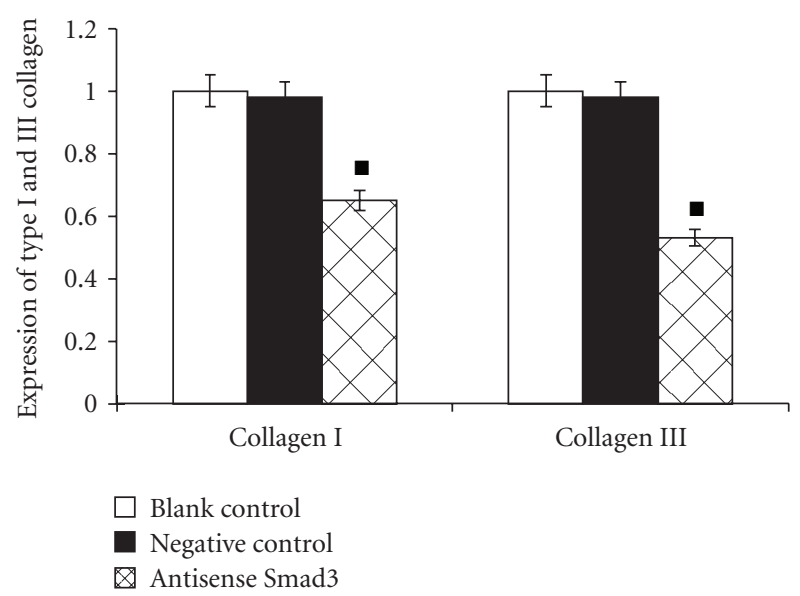

(a)

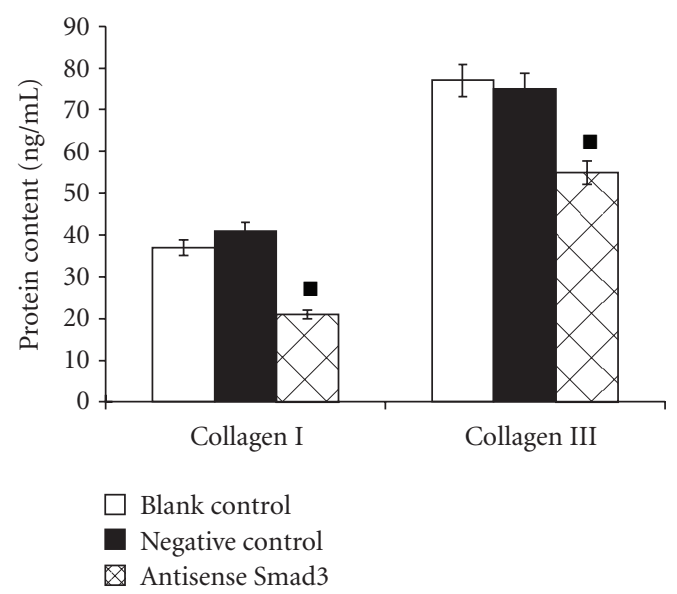

(b)

Figure 3: Expressions of type I and III collagen in VSMCs transfected with adenovirus expressing antisense Smad3 in vitro. Total RNA was extracted for RT-PCR. The culture medium was collected, and ELISA was performed to measure the contents of type I and III collagen. (a) mRNA expression of type I and III collagen in three groups; (b) contents of type I and III collagen in three groups. The mRNA expression of type I and III was normalized to that of $\beta$-actin as the relative expression. Data were expressed as mean \pm SD. ${ }^{\wedge} P<0.05, \quad P<0.01$ versus negative control group.

TGF- $\beta 1$ can increase the phosphorylation of Smad2 and Smad3, which then form heterogeneous trimmers with Smad4. The heterogeneous trimmers then translocate into the nucleus and bind to the Smad-related DNA sequence, leading to the transcription of fibronectin, type I collagen and connective tissue growth factor, which may finally result in vascular RS. Multiple in vivo models [23-26] revealed that the protein expression of Smads was upregulated at the site of arterial injury. Studies on carotid artery injury [27] demonstrated an upregulated expression of Smad3 but not Smad2 in both medial and neointimal cells. In humans, the Smad3 expression at the site of RS was higher than that in the atherosclerotic plaques [28]. The majority of cells expressing Smad3 at high level also express the markers of proliferation. The Smad3 treated artery was found to have larger lumen than that in the control group, which indicates that Smad3 involves in the vascular remodeling [27].

The establishment of carotid artery injury in rats is relatively simple in operation and has relatively high success rate. In addition, the pathophysiology of intimal hyperplasia following injury, and changes in the expression of related cytokines and proteins are similar to those in humans. Thus, this animal model has been widely used in the investigation of postoperative vascular RS [14]. In our previous study [12], the Smad3 expression in the blood vessels was detected at different time points after injury. Results showed the Smad3 expression increased dramatically at 1 day after injury $(P<0.05)$ and the high Smad3 expression maintained for 2 weeks $(P<0.05)$ but returned to nearly normal level at 3 months after injury, which were consistent with previous findings [26]. In the present study, the VSMCs were collected from rats undergoing balloon injury, and results (Figure 1) showed the expressions of TGF- $\beta 1$, Smad 3 , and type I and III collagen were markedly increased when compared with the normal group $(P<0.05)$.

To further investigate the role of TGF- $\beta 1 / \mathrm{Smad} 3$ signaling pathway in the VSMCs induced RS, adenovirus expressing antisense Smad3 was constructed and used to transfect VSMCs from rats with balloon injury and the mRNA expression of $\mathrm{Smad} 3$ was detected. Results demonstrated that the mRNA expression of Smad3 was reduced by about $50 \%$ (Figure 2).

Collagen is the most abundant component of ECM in the vascular wall and accounts for more than $50 \%$ of vascular wall with stenosis. To date, 13 types of collagen have been identified of which 6 were found to be closely related to the vascular wall (I, III, IV, V, VI, and VIII). Among 6 types of collagen, types I, III, and IV have been found to be closely related the vascular RS. Type I and III collagen belongs to the interstitial collagen and accounts for 80 $90 \%$ of collagen in the blood vessels. In the present study, our results showed, in VSMCs transfected with adenovirus expressing antisense Smad3, the TGF- $\beta 1 / \mathrm{Smad} 3$ expression was reduced, and type I and III collagen expression also decreased (Figure 3). Further in vivo study revealed, at 2 weeks and 3 months after injury, the neointima area reduced but the lumen area increased after injection of adenovirus expressing antisense Smad3 (Table 2). These findings suggest that to block the TGF- $\beta 1 / \mathrm{Smad} 3$ signaling pathway may reduce the neointimal hyperplasia to a certain extent, which may attribute to the reduced generation of ECM and type I and III collagen.

Madri et al. [29] first noted increased immunostaining for TGF- $\beta 1$ in the intimal regions of rat carotid arteries 10 weeks after balloon catheter injury. Majesky et al. [30] found that VSMCs had significantly enhanced TGF- $\beta 1$ levels $6 \mathrm{~h}$ after carotid injury in rats, being 5-7-fold above baseline within $24 \mathrm{~h}$, and remaining elevated for 2 weeks as intimal thickening occurred. In porcine coronary arteries [31], balloon angioplasty injury resulted in significantly elevated active TGF- $\beta 1$ levels between $2 \mathrm{~h}$ and 7 days after intervention, returning to baseline 28 days later. Nikol et al. $[5,32]$ found expression of transforming growth factor- $\beta 1$ is increased in human vascular RS lesions. 


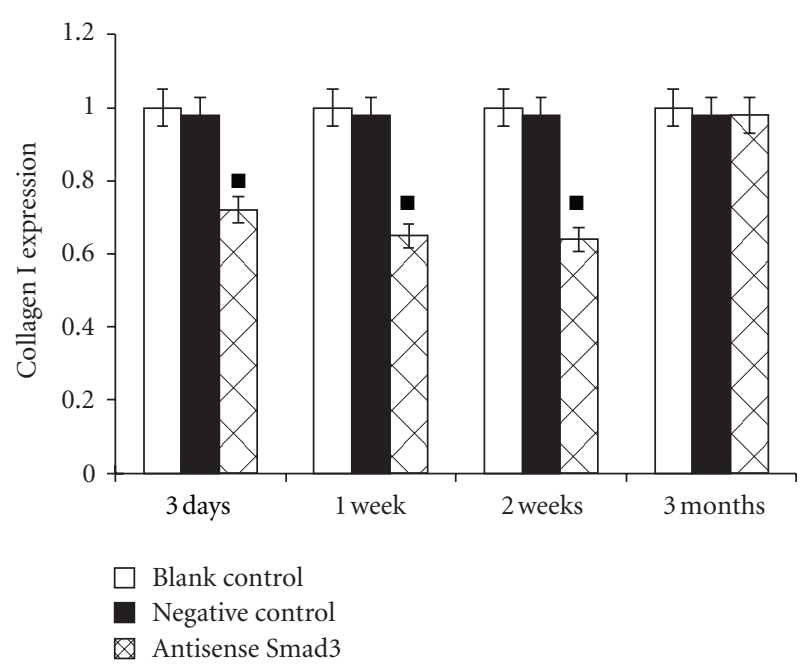

(a)

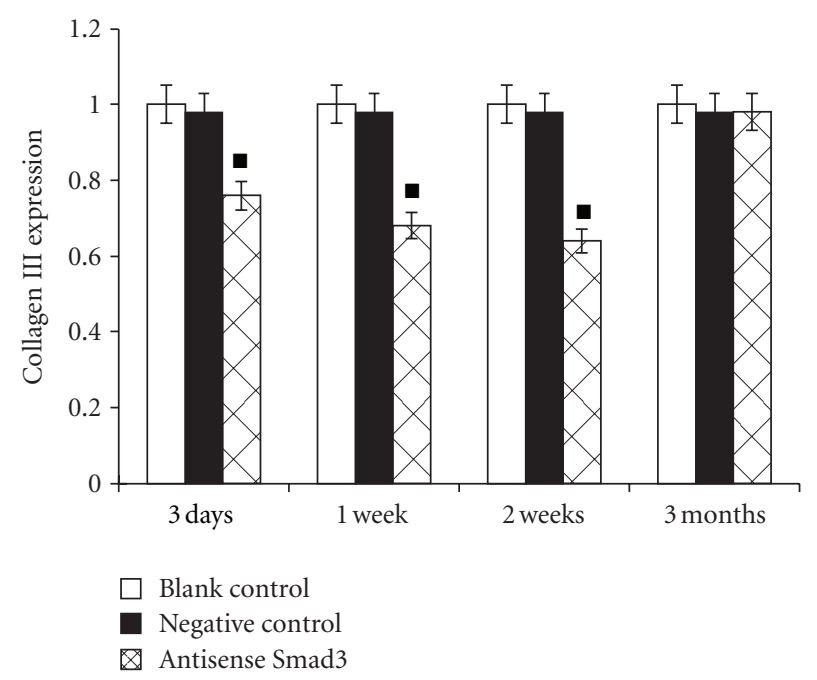

(c)

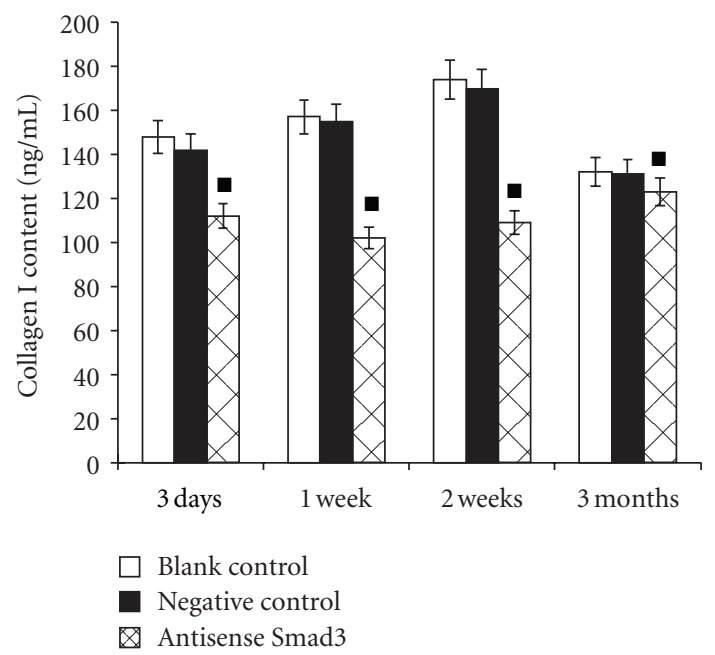

(b)

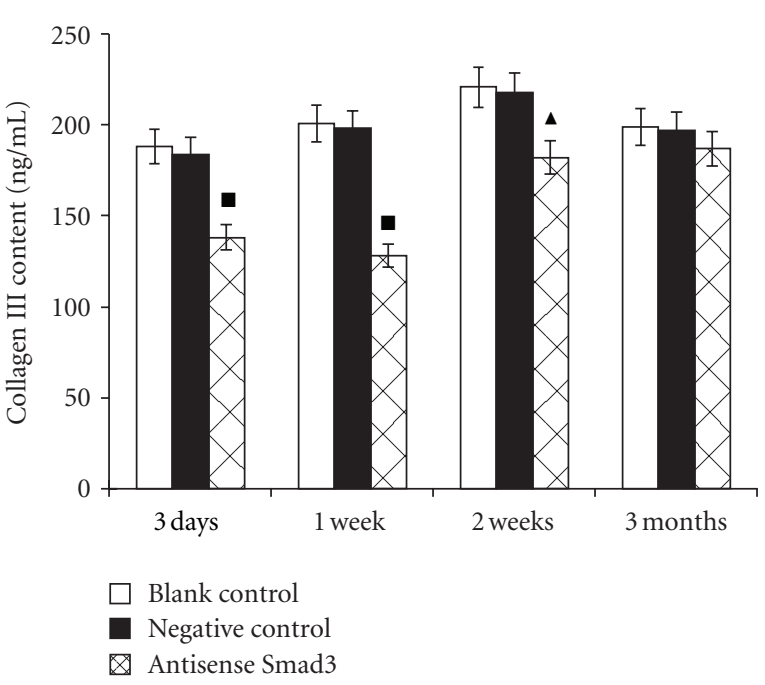

(d)

FIGURE 4: Expressions of type I and III collagen in VSMCs following injection of adenovirus expressing antisense Smad3 in vivo. Ninety SD rats were randomly assigned into blank control group, experiment group, and negative control group. In all groups ( $n=5$ per group), rats were sacrificed at 1 day, 3 days, 1 week, 2 weeks, 1 month, and 3 months after injury followed by sample collection. Total RNA was extracted for the detection of mRNA expressions of type I and III collagen with quantitative PCR (a, c). In addition, the serum was also collected from rats followed by detection of type I and III collagen contents with ELISA (b, d). (a) mRNA expression of type I collagen in different groups; (b) content of type I collagen in the serum of different groups; (c) mRNA expression of type III collagen in different groups; (d) content of type III collagen in the serum of different groups. The mRNA expressions of type I and III collagen were normalized to that of $\beta$-actin. The mRNA expressions of type I and III collagen in the blank control group served as the baseline levels, and those in other groups were normalized to those in the blank control group as the fold change which was used for delineation. Data were expressed as mean \pm SD. $\Delta P<0.05, \quad P<0.01$ versus negative control group.

At early stage of acute injury, the TGF- $\beta 1$ expression is significantly increased, which may be related to the thrombosis in the acute phase and release of a large amount of growth factors by platelets and local inflammation. At early stage of intima injury, the TGF- $\beta 1$ expression increases as a response involving in the repair of blood vessels. At 2 weeks after injury, the serum TGF- $\beta 1$ level decreased to a certain extent, but was still at a high level when compared with the control group. At this time, the TGF- $\beta 1$ further exerts biological effects to promote the proliferation of SMCs and facilitate the deposition of ECM. In the present study, the type I and III collagen was significantly reduced at 3 days and 2 weeks after injury (Figure 4), but the changes in lumen area and intima area were the most evident at 2 weeks and 3 months after injury in the antisense Smad3 group (Table 2).

Our findings suggest that the transfection with adenovirus expressing antisense Smad3 can inhibit the Smad3 expression, which then suppresses the collagen metabolism, reduces the ECM deposition, and inhibits the neointimal proliferation to a certain extent. Our findings provide evidence for the gene therapy of RS following PTCA. 


\section{References}

[1] C. Giglioli, S. Valente, M. Margheri et al., "An angiographic evaluation of restenosis rate at a six-month follow-up of patients with ST-elevation myocardial infarction submitted to primary percutaneous coronary intervention," International Journal of Cardiology, vol. 131, no. 3, pp. 362-369, 2009.

[2] A. B. Osherov, L. Gotha, A. N. Cheema, B. Qiang, and B. H. Strauss, "Proteins mediating collagen biosynthesis and accumulation in arterial repair: novel targets for antirestenosis therapy," Cardiovascular Research, vol. 91, no. 1, pp. 16-26, 2011.

[3] A. Curcio, D. Torella, and C. Indolfi, "Mechanisms of smooth muscle cell proliferation and endothelial regeneration after vascular injury and stenting," Circulation Journal, vol. 75, no. 6, pp. 1287-1296, 2011.

[4] R. Khan, A. Agrotis, and A. Bobik, "Understanding the role of transforming growth factor- $\beta 1$ in intimal thickening after vascular injury," Cardiovascular Research, vol. 74, no. 2, pp. 223-234, 2007.

[5] S. T. Ryan, V. E. Koteliansky, P. J. Gotwals, and V. Lindner, "Transforming growth factor-beta-dependent events in vascular remodeling following arterial injury," Journal of Vascular Research, vol. 40, no. 1, pp. 37-46, 2003.

[6] A. Bobik, "Transforming growth factor-betas and vascular disorders," Arteriosclerosis, Thrombosis, and Vascular Biology, vol. 26, pp. 1712-1720, 2006.

[7] T. Kanzaki, K. Tamura, K. Takahashi et al., "In vivo effect of TGF- $\beta 1$ : enhanced intimal thickening by administration of TGF- $\beta 1$ in rabbit arteries injured with a balloon catheter," Arteriosclerosis, Thrombosis, and Vascular Biology, vol. 15, no. 11, pp. 1951-1957, 1995.

[8] M. R. Ward, A. Agrotis, P. Kanellakis, J. Hall, G. Jennings, and A. Bobik, "Tranilast prevents activation of transforming growth factor- $\beta$ system, leukocyte accumulation, and neointimal growth in porcine coronary arteries after stenting," Arteriosclerosis, Thrombosis, and Vascular Biology, vol. 22, no. 6, pp. 940-948, 2002.

[9] M. J. Merrilees, B. Beaumont, L. Scott, V. Hermanutz, and P. Fennessy, "Effect of TGF- $\beta 1$ antisense S-oligonucleotide on synthesis and accumulation of matrix proteoglycans in balloon catheter-injured neointima of rabbit carotid arteries," Journal of Vascular Research, vol. 37, no. 1, pp. 50-60, 2000.

[10] E. J. Ryer, R. P. Hom, K. Sakakibara et al., "PKC $\delta$ is necessary for Smad3 expression and transforming growth factor $\beta$ induced fibronectin synthesis in vascular smooth muscle cells," Arteriosclerosis, Thrombosis, and Vascular Biology, vol. 26, no. 4, pp. 780-786, 2006.

[11] Z. H. Cheng, W. W. Cai, P. Lu, S. J. Ma, Y. Chen, and J. Sheng, "Effects of signal transduction interruption of transforming growth factor- $\beta 1$ by anti-Smad3 on proliferation of vascular smooth muscle cells," Journal of Shanghai Jiaotong University, vol. 29, no. 8, pp. 935-937, 2009.

[12] P. Lu, S. H. Wang, W. W. Cai, and J. Sheng, "TGF-beta 1/Smad3 expression and its effects on carotid intimal hyperplasia in balloon-injured rats," Frontiers in Bioscience, vol. 4, pp. 20222028, 2012.

[13] J. J. Wu, D. R. Wang, J. H. Lu et al., "Construction of recombinant antisense Smad3 adenoviral vector and its expression in vitro," Journal of Shanghai Second Medical University, vol. 24, no. 8, pp. 600-602, 2004.

[14] J. M. Li, X. Zhang, P. R. Nelson et al., “Temporal evolution of gene expression in rat carotid artery following balloon angioplasty," Journal of Cellular Biochemistry, vol. 101, no. 2, pp. 399-410, 2007.

[15] G. Dangas and V. Fuster, "Management of restenosis after coronary intervention," American Heart Journal, vol. 132, no. 2 I, pp. 428-436, 1996.

[16] R. S. Schwartz, D. R. Holmes, and E. J. Topol, "The restenosis paradigm revisites: an alternative proposal for cellular mechanisms," Journal of the American College of Cardiology, vol. 20, no. 5, pp. 1284-1293, 1992.

[17] A. Backes, U. Seay, D. G. Sedding, H. H. Tillmanns, and R. C. Braun-Dullaeus, "Inhibition of matrix deposition: a new strategy for prevention of restenosis after balloon angioplasty," Journal of Cardiovascular Pharmacology, vol. 55, no. 2, pp. 213-218, 2010.

[18] M. Ruiz-Ortega, J. Rodríguez-Vita, E. Sanchez-Lopez, G. Carvajal, and J. Egido, "TGF- $\beta$ signaling in vascular fibrosis," Cardiovascular Research, vol. 74, no. 2, pp. 196-206, 2007.

[19] K. Yokote, K. Kobayashi, and Y. Saito, "The role of Smad3dependent TGF- $\beta$ signal in vascular response to injury," Trends in Cardiovascular Medicine, vol. 16, no. 7, pp. 240-245, 2006.

[20] M. Wildgruber, W. Weiss, H. Berger, O. Wolf, H. H. Eckstein, and P. Heider, "Association of circulating transforming growth factor beta, tumor necrosis factor alpha and basic fibroblast growth factor with restenosis after transluminal angioplasty," European Journal of Vascular and Endovascular Surgery, vol. 34, no. 1, pp. 35-43, 2007.

[21] H. Y. Zhou, W. D. Chen, D. L. Zhu et al., "The PDE1APKCalphasignaling pathway is involved in the up regulation of alpha-smooth muscle actin by TGF-beta1 in adventitial fihroblasts," Journal of Vascular Research, vol. 47, pp. 9-15, 2010.

[22] J. Yu, L. Zhang, A. Chen et al., "Identification of the gene transcription and apoptosis mediated by TGF- $\beta$-Smad2/3Smad4 signaling," Journal of Cellular Physiology, vol. 215, no. 2, pp. 422-433, 2008.

[23] J. P. G. Sluijter, R. E. Verloop, W. P. C. Pulskens et al., "Involvement of furin-like proprotein convertases in the arterial response to injury," Cardiovascular Research, vol. 68, no. 1, pp. 136-143, 2005.

[24] R. J. LeClair, T. Durmus, Q. Wang, P. Pyagay, A. Terzic, and V. Lindner, "Cthrc1 is a novel inhibitor of transforming growth factor- $\beta$ signaling and neointimal lesion formation," Circulation Research, vol. 100, no. 6, pp. 826-833, 2007.

[25] W. Zeng, W. Chen, X. Leng, J. G. He, and H. Ma, "Chronic angiotensin-(1-7) administration improves vascular remodeling after angioplasty through the regulation of the TGF- $\beta$ /Smad signaling pathway in rabbits," Biochemical and Biophysical Research Communications, vol. 389, no. 1, pp. 138144, 2009.

[26] S. Tsai, S. T. Hollenbeck, E. J. Ryer et al., "TGF- $\beta$ through Smad3 signaling stimulates vascular smooth muscle cell proliferation and neointimal formation," American Journal of Physiology, vol. 297, no. 2, pp. H540-H549, 2009.

[27] R. Kundi, S. T. Hollenbeck, D. Yamanouchi et al., "Arterial gene transfer of the TGF- $\beta$ signalling protein Smad3 induces adaptive remodelling following angioplasty: a role for CTGF," Cardiovascular Research, vol. 84, no. 2, pp. 326-335, 2009.

[28] R. S. Edlin, S. Tsai, D. Yamanouchi, C. Wang, B. Liu, and K. C. Kent, "Characterization of primary and restenotic atherosclerotic plaque from the superficial femoral artery: potential role of Smad3 in regulation of SMC proliferation," Journal of Vascular Surgery, vol. 49, no. 5, pp. 1289-1295, 2009. 
[29] J. A. Madri, M. A. Reidy, O. Kocher, and L. Bell, "Endothelial cell behavior after denudation injury is modulated by transforming growth factor- $\beta 1$ and fibronectin," Laboratory Investigation, vol. 60, no. 6, pp. 755-765, 1989.

[30] M. W. Majesky, V. Lindner, D. R. Twardzik, S. M. Schwartz, and M. A. Reidy, "Production of transforming growth factor $\beta 1$ during repair of arterial injury," Journal of Clinical Investigation, vol. 88, no. 3, pp. 904-910, 1991.

[31] J. Chamberlain, J. Gunn, S. E. Francis et al., "TGF $\beta$ is active, and correlates with activators of TGF $\beta$, following porcine coronary angioplasty," Cardiovascular Research, vol. 50, no. 1, pp. 125-136, 2001.

[32] S. Nikol, J. M. Isner, J. G. Pickering, M. Kearney, G. Leclerc, and L. Weir, "Expression of transforming growth factor- $\beta 1$ is increased in human vascular restenosis lesions," Journal of Clinical Investigation, vol. 90, no. 4, pp. 1582-1592, 1992. 

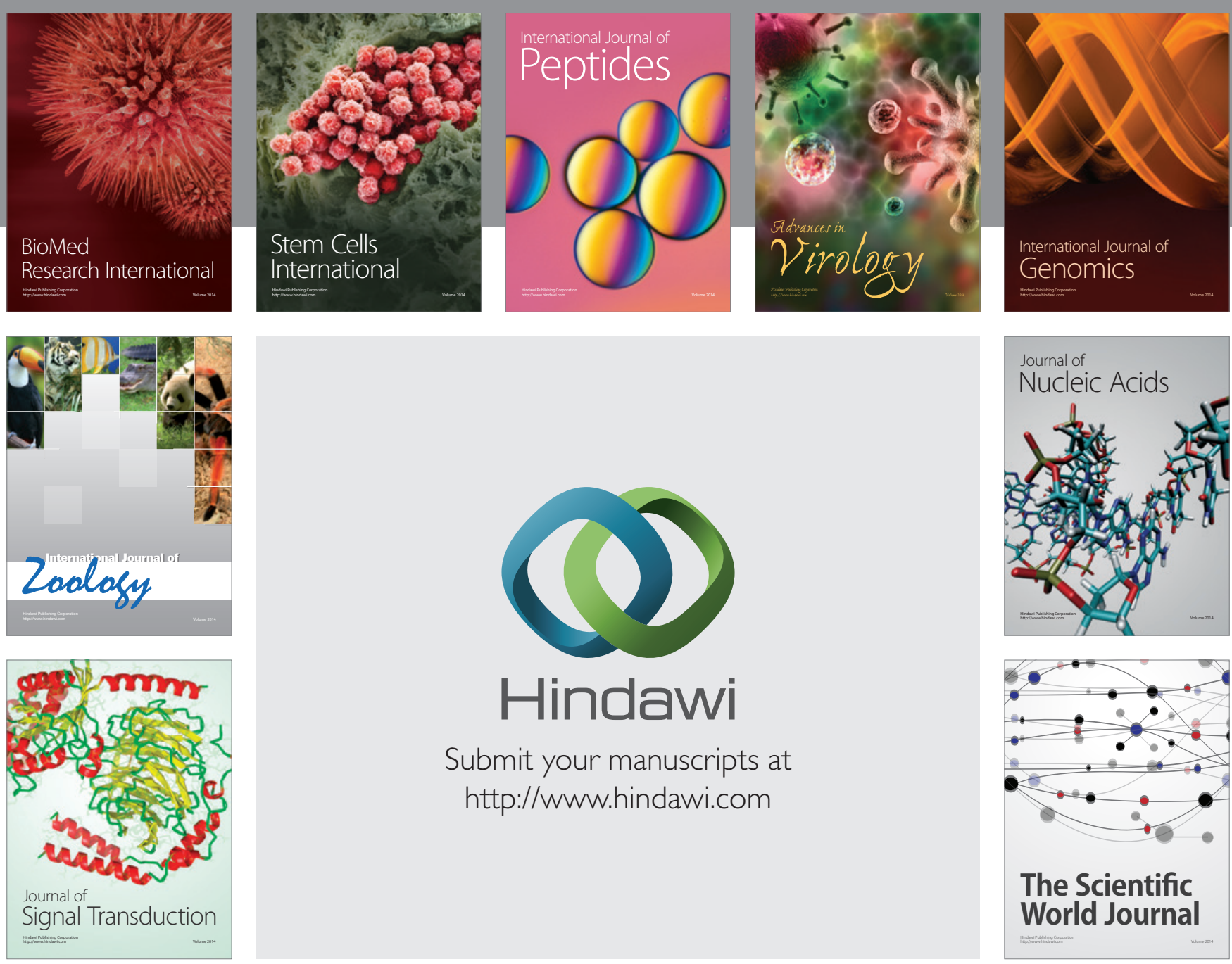

Submit your manuscripts at

http://www.hindawi.com
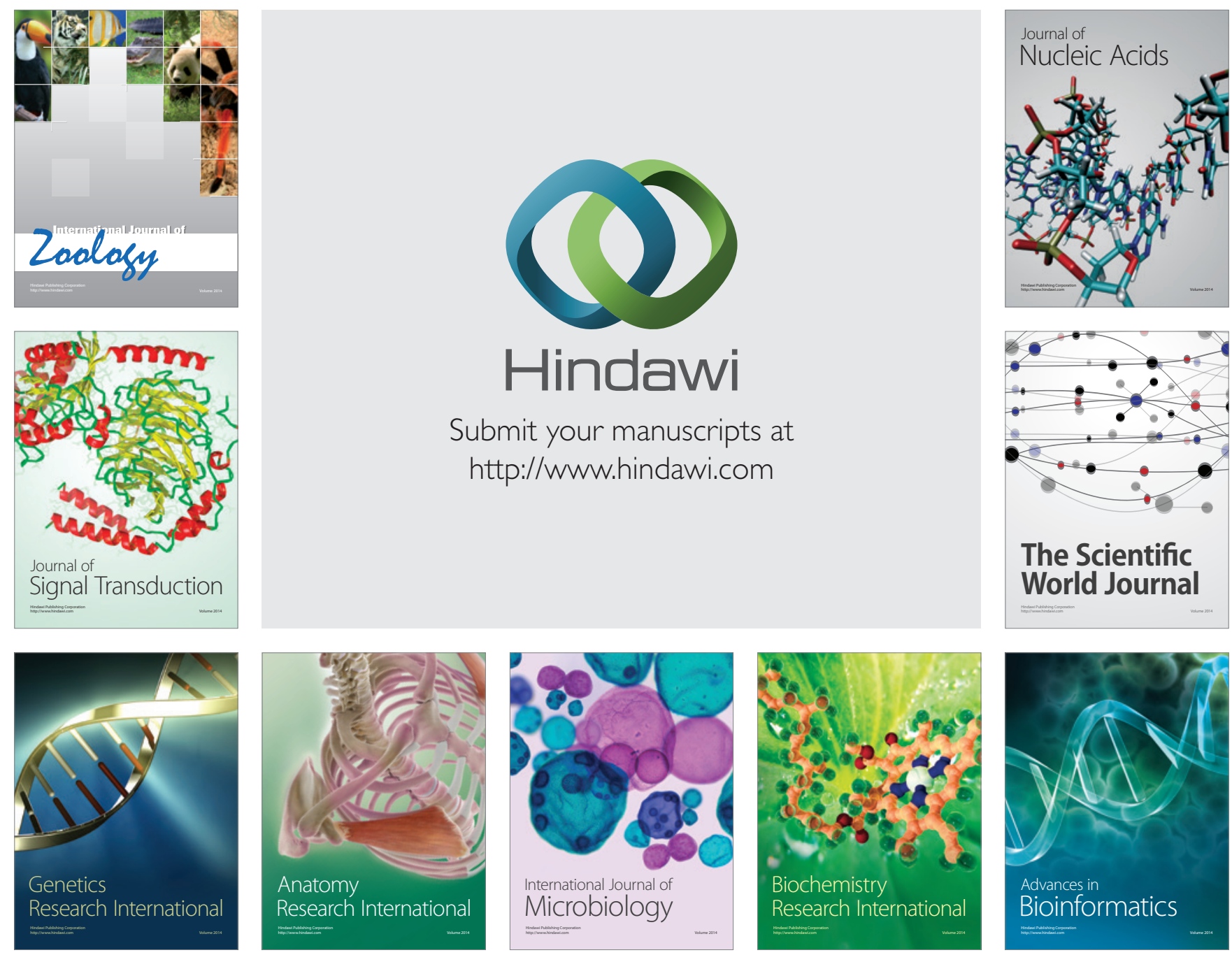

The Scientific World Journal
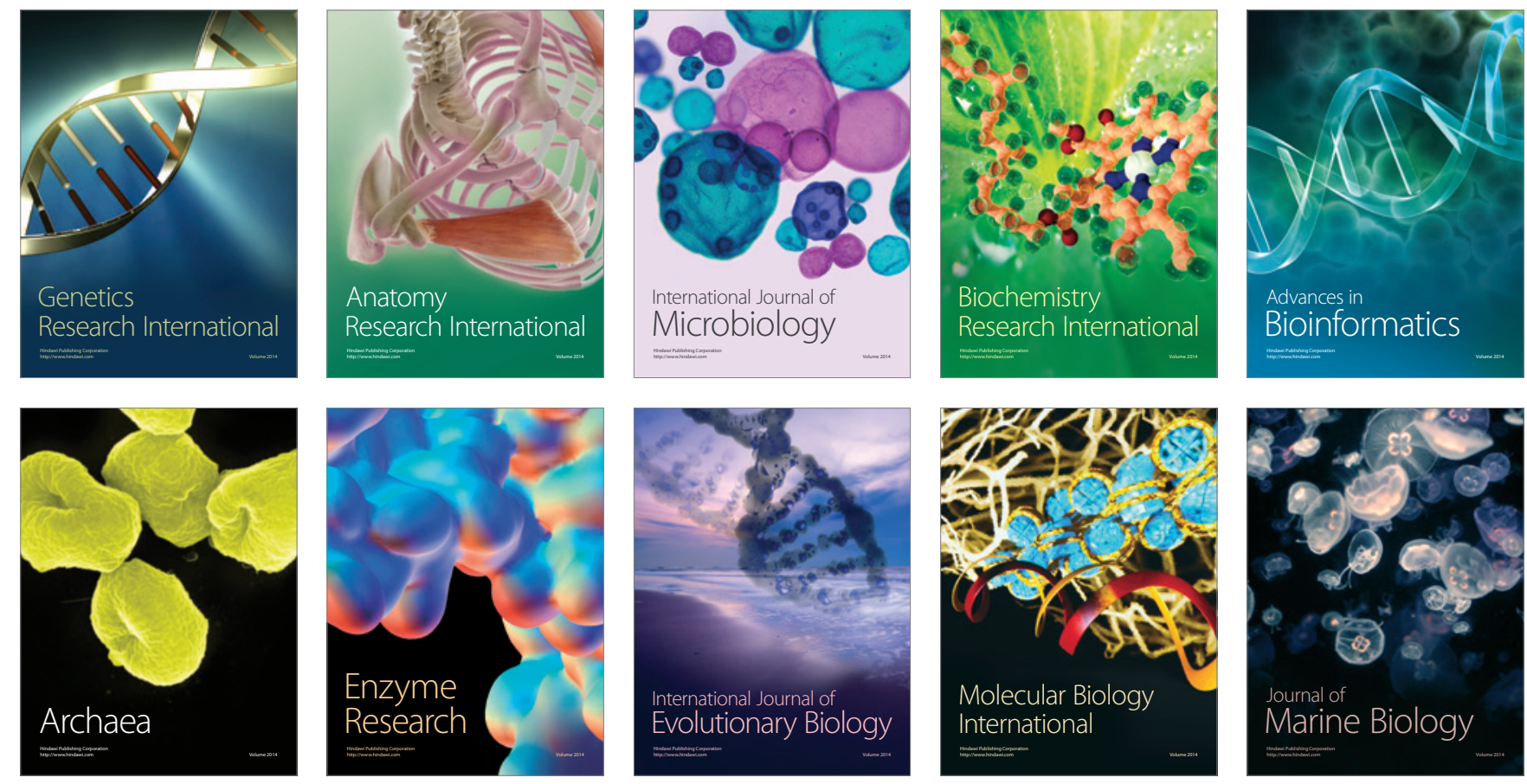